\title{
INVERSE SPECTRAL NONLOCAL PROBLEM FOR THE FIRST ORDER ORDINARY DIFFERENTIAL EQUATION
}

\author{
LEONID NIZHNIK
}

\begin{abstract}
We solve direct and inverse spectral problems for the first order ordinary differential equation with nonlocal potential and nonlocal boundary condition.
\end{abstract}

\section{Introduction}

An inverse spectral problem for some nonlocal operators have been studied in [1]-[6]. A Schrödinger operator with a nonlocal point potential was introduced and studied in [7]. Direct and inverse nonlocal problems for the Sturm-Liouville operator with various boundary conditions were solved in [8]-[10].

In this paper, we study inverse spectral nonlocal problems for the first order ordinary differential equation with a nonlocal potential and a nonlocal boundary condition. Then we consider the following nonlocal eigenvalue problem:

$$
i y^{\prime}(x)+v(x) y_{-}=\lambda y(x), \quad x \in(-\pi, \pi),
$$

subject to the boundary-value condition

$$
y_{+}+2 i(y, v)_{L_{2}}=0
$$

Here $v \in L_{2}(-\pi, \pi)$ is a nonlocal "potential", $\lambda \in \mathbb{C}$ is a spectral parameter and $y_{-}=y(\pi)-$ $y(-\pi), y_{+}=y(\pi)+y(-\pi)$ are boundary data for the function $y(x)$.

The problem (1)-(2) is self-adjoint in the space $L_{2}(-\pi, \pi)$. This follows from the following analogue of Lagrange's formula, which holds for arbitrary functions $y, \varphi$ that belong to the space $W_{2}^{1}(-\pi, \pi)$ :

$$
\left(i y^{\prime}+v y_{-}, \varphi\right)_{L_{2}}-\left(y, i \varphi^{\prime}+v \varphi_{-}\right)_{L_{2}}=-\frac{i}{2}\left[y_{+}+2 i(y, v)_{L_{2}}\right] \bar{\varphi}_{-}+\frac{i}{2} y_{-}\left[\bar{\varphi}_{+}-2 i(v, \varphi)_{L_{2}}\right] .
$$

2000 Mathematics Subject Classification. Primary 34A55; Secondary 34B24, 34L05, 47E05.

Key words and phrases. Spectrum, eigenvalue, inverse spectral problem, nonlocal boundary conditions. 


\section{Spectral problem}

Spectral problem (1), (2) is naturally related to an operator $T$ on the Hilbert space $H=$ $L_{2}(-\pi, \pi)$. It has domain

$$
\operatorname{dom} T=\left\{y \mid y \in W_{2}^{1}(-\pi, \pi), y \text { satisfies boundary condition (2) }\right\}
$$

and is defined by $T y=i y^{\prime}(x)+v(x) y_{-}$. The operator $T$ is a self-adjoint operator on $H$. The self-adjoint operator $T$ is a finite rank perturbation of a self-adjoint operator $T_{p}$ defined by $T_{p} y=i y^{\prime}(x)$ on functions that satisfy the boundary conditions $y(\pi)=y(-\pi)$.

Since the operator $T_{p}$ has discrete spectrum, which consists of the eigenvalues $\lambda_{n}=n$, $n \in Z$, the operator $T$ has discrete spectrum consisting of real numbers $\lambda_{n} \rightarrow+\infty$ and $\lambda_{-n} \rightarrow$ $-\infty$ for $n \rightarrow \infty$.

Theorem 1. All eigenvalues of problem (1), (2), distinct from $n, n \in Z$, are real and simple.

The number $\lambda=n$ is an eigenvalue of problem (1), (2) if and only if

$$
\nu_{-n}=\frac{1}{2 \pi} \int_{-\pi}^{\pi} v(x) e^{i n x} d x=\frac{i}{2 \pi}(-1)^{n+1}
$$

The number $\lambda=n$ is a double eigenvalue of problem (1), (2) if and only if, in addition to (4), we have

$$
\sum_{k+n \neq 0} \frac{1}{k+n}\left[\left(v_{k}-\bar{v}_{k}\right)(-1)^{k}+2 \pi i\left|v_{k}\right|^{2}\right]=0
$$

Problem (1), (2) has no eigenvalues with multiplicity exceeding 2.

Proof. The eigenvalues of problem (1), (2) are real, since they are eigenvalues of the selfadjoint operator $T$.

If an eigenvalue $\lambda_{0}$ were twofold, then there would exist two linearly independent eigenfunctions $y_{1}$ and $y_{2}$ corresponding to this eigenvalue. Since any linear combinations $y=$ $C_{1} y_{1}+C_{2} y_{2}$ is also an eigenfunctions of problem (1), (2) corresponding to the eigenvalue $\lambda_{0}$, by choosing $C_{1}=y_{2-}$ and $C_{2}=-y_{1-}$, we get $y_{-}=0$. If $y \equiv 0$, then $y_{1-}=y_{2_{-}}=0$. Hence, for the eigenvalue $\lambda_{0}$ there exists a nonzero eigenfunction $y$ that satisfies the boundary condition $y_{-}=0$. It follows from (1) that $y=e^{-i \lambda_{0} x}$. The condition $y_{-}=0$ shows that $\lambda_{0}$ is an integer. Let now $\lambda=n$ be an eigenvalue of problem (1), (2). If the corresponding eigenfunction $y(x ; n)$ assumes equal values at the endpoints of the interval, that is, $y_{-}=0$, then (1) yields $y(x ; n)=e^{-i n x}$, and boundary condition (2) leads to (4). Conversely, assuming (4) shows that the function $y(x ; n)=e^{-i n x}$ is an eigenfunction of problem (1), (2) corresponding to the eigenvalue $\lambda=n$. If condition (4) holds, then equation (1) has a solution distinct from $y(x ; n)=e^{-i n x}$,

$$
\varphi(x ; n)=x e^{-i n x}+2 \pi(-1)^{n} \sum_{k+n \neq 0} \frac{v_{k} e^{-i k x}}{k+n}
$$


where $v_{k}$ are Fourier coefficients of the potential $\nu(x)=\sum_{k=-\infty}^{\infty} v_{k} e^{i k x}$. A function $\varphi(x ; n)$ is an eigenfunction of problem (1), (2) if and only if it satisfies boundary condition (2). This leads to relation (5). The eigenvalue $\lambda=n$ can not have multiplicity exceeding 2 , since equation (1) does not have more than two linearly independent solutions.

To give an exact description of the distribution of eigenvalues of problem (1), (2), it is convenient to show that the eigenvalues are connected with zeros of an analytic function, which is the characteristic function of problem (1), (2).

To this end, consider a special solution of equation (1) with $\lambda=z$,

$$
\varphi(x ; z)=\frac{1}{2} e^{-i z x}-i \sin \pi z \sum_{n=-\infty}^{\infty} \frac{v_{n} e^{i n x}}{n+z} .
$$

The function $\varphi$ is an eigenfunction of problem (1), (2) if it satisfies the boundary-value condition (2). This gives the characteristic equation $\chi(z)=0$, where the characteristic function $\chi(z)$ is defined by $\chi(z)=\varphi_{+}+2 i(\varphi, v)$ and has the form

$$
\chi(z)=\cos \pi z+2 \sin \pi z \sum_{n=-\infty}^{\infty} \frac{\alpha_{n}(-1)^{n}}{n+z},
$$

where

$$
\alpha_{n}=-i v_{n}+i \bar{v}_{n}+2 \pi(-1)^{n}\left|v_{n}\right|^{2} .
$$

Lemma 1. The characteristic function $\chi(z)$ of problem (1),(2) is an entire function of $z$ and takes the values

$$
\chi(-m)=(-1)^{m}\left[1+2 \pi(-1)^{m} \alpha_{m}\right]=(-1)^{m}\left|1-2 \pi i(-1)^{m} v_{m}\right|^{2} .
$$

Proof. The proof is carried out by making direct computations using the explicit form (8) of the characteristic function.

Theorem 2. The number $\lambda$ is an eigenvalue of problem (1), (2) if and only if $\lambda$ is a zero of the characteristic function $\chi(z)$. A number $\lambda$ is a double eigenvalue of the problem (1), (2) if and only if $\lambda$ is a double zero of the characteristic function. All zeros $z \neq n, n \in Z$, of the characteristic function are simple. The characteristic function does not have zeros of multiplicities greater than 2.

Proof. It follows that zeros $\lambda \neq n$ of the characteristic function $\chi(z)$ are eigenvalues, since the special solution (7) is an eigenfunction and vice versa. The number $\lambda=n$ is a eigenvalue if and only if (4) is satisfied which, by (10), is equivalent to $\chi(n)=0$. If the characteristic function $\chi$ had a multiple root $z=n$, this would imply that $\left.\frac{d \chi(z)}{d z}\right|_{z=n}=0$, which is equivalent to (5). The 
characteristic function does not have zeros of multiplicities greater than 2 for, otherwise, the function $\left.\frac{d^{2} \varphi(x ; z)}{d z^{2}}\right|_{z=z_{0}}$ would be a nontrivial generalized eigenfunction for problem (1)-(2), which is impossible since problem (1), (2) is self-adjoint.

\section{Resolvent}

A fundamental solution of the equation $\left(i \frac{d}{d x}-z\right) E=\delta(x-s)$ has the form

$$
E(x-s ; z)=\frac{i}{2} e^{i z(s-x)} \operatorname{sign}(s-x) .
$$

Green's function for the equation $\left(i \frac{d}{d x}-z\right) \mathscr{G}_{p}=\delta(x-s)$ and which corresponds to the periodic boundary condition $y(\pi)=y(-\pi)$ is

$$
\mathscr{G}_{p}(x, s ; z)=E(x-s ; z)-\frac{\cos z \pi}{2 \sin z \pi} e^{-i z x} e^{i z s} .
$$

This means that the resolvent $\left(T_{p}-z I\right)^{-1}$ of the self-adjoint operator $T_{p}$ with periodic boundary conditions is the integral operator $\left(T_{p}-z I\right)^{-1} u(x)=\int_{-\pi}^{\pi} \mathscr{G}_{p}(x, s ; z) u(s) d s$. Green's function $\mathscr{G}_{a}$ for the antiperiodic boundary conditions $y(\pi)=-y(-\pi)$ has the form

$$
\mathscr{G}_{a}(x, s ; z)=E(x-s ; z)+\frac{\sin z \pi}{2 \cos z \pi} e^{-i z x} e^{i z s} .
$$

The function $\mathscr{G}_{a}$ is the kernel of the integral resolvent operator $\left(T_{a}-z I\right)^{-1}$ for the self-adjoint operator $T_{a}$ corresponding to the antiperiodic boundary conditions. It follows from (12)-(13) that

$$
\mathscr{G}_{a}-\mathscr{G}_{p}=\frac{1}{2 \sin 2 \pi z} e^{-i z x} e^{i z s} .
$$

Hence, the integral operators $\left(T_{a}-z I\right)^{-1}$ and $\left(T_{p}-z I\right)^{-1}$ differ by a one-dimensional operator. The self-adjoint operators $T_{a}$ and $T_{p}$ are rank one perturbations of each other.

Simple calculations show that the resolvent $(T-z I)^{-1}$ of the main operator for problem (1)-(2) is an integral operator whose kernel is Green's function

$$
\mathscr{G}(x, s ; z)=\mathscr{G}_{p}(x, s ; z)+\frac{2}{\sin \pi z \chi(z)} \varphi(x ; z) \bar{\varphi}(s ; \bar{z}) .
$$

Formula (15) shows that the self-adjoint operator $T$ is a rank one perturbation of the self-adjoint operator $T_{p}$, and (14) with $v \neq 0$ shows that it is a rank two perturbation of the self-adjoint operator $T_{a}$. Note that, if $v=0$, then $T=T_{a}$. 


\section{Example of a constant potential}

Consider the case where the nonlocal potential is a pure imaginary constant, $v(x) \equiv i \beta$. Here, the characteristic equation is

$$
\chi(\lambda)=\cos \lambda \pi-\gamma \frac{\sin \lambda \pi}{\lambda \pi}=0,
$$

where $\gamma=-4 \pi \beta(1+\pi \beta)$ is a real number. Since the function $\chi(\lambda)$ is even, the set of its zeros is symmetric, $\lambda_{n}=-\lambda_{-n}, n \in N$. We have $\lambda_{1}=\lambda_{-1}=0$ if and only if $\gamma=1$, that is, $\beta=-\frac{1}{2 \pi}$. Eigenfunctions have the form

$$
\varphi\left(x ; \lambda_{n}\right)=\frac{1}{2} e^{-i \lambda_{n} x}+\beta \frac{\sin \lambda_{n} \pi}{\lambda_{n}}, n= \pm 1, \pm 2, \ldots
$$

They form a complete orthonormal system in the space $L_{2}(-\pi, \pi)$, being a complete system of eigenfunctions of a self-adjoint operator $T$. By passing to the functions $s_{n}(x)=i\left[\varphi\left(x ; \lambda_{n}\right)-\right.$ $\left.\varphi\left(x ;-\lambda_{n}\right)\right]=\sin \lambda_{n} x$ and $c_{n}(x)=\varphi\left(x ; \lambda_{n}\right)+\varphi\left(x ;-\lambda_{n}\right)=\cos \lambda_{n} x+\delta_{n}$, where $\delta_{n}=-\frac{\cos \lambda_{n} \pi}{2(1+\pi \beta)}$, we obtain a complete orthonormal system of functions in the space $L_{2}(-\pi, \pi)$,

$$
s_{n}(x)=\sin \lambda_{n} x, \quad c_{n}(x)=\cos \lambda_{n} x+\delta_{n},
$$

where $\lambda_{n} \geq 0, n \in N$, are roots of the characteristic equation (16). Note that the functions in system (18) can be connected to eigenfunctions of usual self-adjoint boundary problems. Indeed, if $x \geq 0$, the functions $s_{n}(x)$ are eigenfunctions of the self-adjoint boundary problem $y^{\prime \prime}+\lambda^{2} y=0$ on the interval $(0, \pi)$ with the boundary conditions $y(0)=0, y^{\prime}(\pi)-\frac{\gamma}{\pi} y(\pi)=0$. Since the functions $s_{n}(x)$ are odd, they can be considered on the interval $[-\pi, \pi]$. We have $c_{n}(x)=\frac{1}{\lambda_{n}} s_{n}^{\prime}(x)+\delta_{n}$, where the constants $\delta_{n}$ are uniquely determined by the condition that the system $\left\{c_{n}(x)\right\}_{n=1}^{\infty}$ is orthogonal in the space $L_{2}(-\pi, \pi)$.

\section{Distribution of the eigenvalues}

In the definition (8)-(9) of the characteristic function, the values $\alpha_{n}$ can be regarded as Fourier coefficients of the function $\alpha(x) \in L_{2}(-\pi, \pi), \alpha(x)=\sum_{n=-\infty}^{\infty} \alpha_{n} e^{i n x}$. Then the characteristic function $\chi$ admits, in addition to representation (8), the representation

$$
\chi(z)=\cos \pi z+\int_{-\pi}^{\pi} e^{i z t} \alpha(t) d t,
$$

too. Representation (19) for the characteristic function $\chi$ permits to recover the function $\chi$ from the set of its zeros. We use the following analogue of a result due to Marchenko, see [11], Lemma 3.4.2. 
Lemma 2. For a function $\chi(z)$ to admit the representation

$$
\chi(z)=\cos \pi z+\int_{-\pi}^{\pi} e^{i z t} \alpha(t) d t
$$

where $\alpha(t) \in L_{2}(-\pi, \pi)$, it is necessary and sufficient that

$$
\begin{aligned}
\chi(z) & =\prod_{k=1}^{\infty}\left(k-\frac{1}{2}\right)^{2}\left(a_{k}-z\right)\left(z-a_{-k}\right), \\
a_{k} & =k-\frac{1}{2} \operatorname{sign} k+\beta_{k},
\end{aligned}
$$

where $\beta_{k}$ is an arbitrary number sequence satisfying the condition

$$
\sum_{k=-\infty}^{\infty}\left|\beta_{k}\right|^{2}<+\infty
$$

Theorem 3. An increasingly ordered two-sided sequence

$$
\cdots \leq \lambda_{-n} \cdots \leq \lambda_{-2} \leq \lambda_{-1} \leq \lambda_{1} \leq \lambda_{2} \leq \ldots \leq \lambda_{n} \leq \ldots
$$

is a sequence of all eigenvalues of problem (1), (2), counting multiplicities, if and only if it has the following properties:

1. the sequence weakly alternates with the sequence $n$,

$$
n-1 \leq \lambda_{n} \leq n, \quad-n \leq \lambda_{-n} \leq-n+1, \quad n \in N
$$

2. there is an asymptotic representation,

$$
\begin{aligned}
& \lambda_{n}=n-\frac{1}{2}+\beta_{n}, \\
& \lambda_{-n}=-n+\frac{1}{2}+\beta_{-n}, \quad n \in N,
\end{aligned} \quad \text { where } \quad \sum_{j=-\infty}^{\infty} \beta_{j}^{2}<+\infty .
$$

Proof. Let $\cdots \leq \lambda_{-n} \cdots \leq \lambda_{-2} \leq \lambda_{-1} \leq \lambda_{1} \leq \lambda_{2} \leq \ldots \leq \lambda_{n} \leq \ldots$ be a sequence of eigenvalues of problem (1), (2) and $\chi(z)$ be its characteristic function.

By the Rouché theorem, the entire function $\chi(z)$ and the function $\cos \pi z$ have the same number of zeros, counting multiplicities, in the strip $|\operatorname{Re} z|<n$ for large $n$. Since, by Theorem 2, the eigenvalues $\lambda_{n}$ of problem (1), (2) are zeros of the function $\chi(z)$ and zeros of the function $\cos \pi z$ are the numbers $z_{n}=n-\frac{1}{2}, z_{-n}=-n+\frac{1}{2}, n \in N$, we see that the inequalities $n<\lambda_{n+1}, \lambda_{-n-1}<-n$ hold for large $n$.

On the other hand, $\chi(n) \neq 0$ for any $n \in Z$ and, moreover, $\chi(n)(-1)^{n}>0$. Hence, in every interval $I_{n}=(n, n+1)$, the characteristic function $\chi$ has at least one zero $z_{0}$ and, consequently, there is one eigenvalue $\lambda=z_{0}$ in the interval $I_{n}$. The assumption that at least one interval $I_{n}$ 
contains more than one eigenvalue leads to a contradiction with the estimate for $\lambda_{n}$ for large $|n|$. Hence, if the conditions $\chi(n) \neq 0$ are satisfied, inequality (23) holds,

$$
n-1<\lambda_{n}<n, \quad-n<\lambda_{-n}<-n+1 .
$$

Since the condition $\chi(n) \neq 0$ can be satisfied by an arbitrary small change of the potential, passing to the limit in (25) we get (23). The asymptotic representations (24) follows from the asymptotic representation (21) of zeros of the characteristic function $\chi(z)$, since $\lambda_{k}=z_{k}$.

Let us now prove that conditions (23)-(24) are sufficient.

Let a sequence $\ldots \leq \lambda_{-n} \ldots \leq \lambda_{-2} \leq \lambda_{-1} \leq \lambda_{1} \leq \lambda_{2} \leq \ldots \leq \lambda_{n} \leq \ldots$ satisfy the conditions (23) and (24). Then the numbers $a_{k}=\lambda_{k}$ satisfy conditions (21) and, by Lemma 2 , there is a function $\alpha \in L_{2}(-\pi, \pi)$ such that representation (21) holds.

Since all $a_{n}=\lambda_{n}$ are real, it follows from (21)-(23) that $(-1)^{n} \chi(n) \geq 0$. This permits, by solving equation (10), to construct $v_{k}$ from the Fourier coefficients $\alpha_{k}$ of the function $\alpha \in L_{2}$.

Construct a function $v \in L_{2}(-\pi, \pi)$ from its Fourier coefficients $v_{n}$. Then the function $\chi(z)$ will be a characteristic function for problem (1), (2) with the above constructed potentials, and the numbers $\lambda_{k}$ make a set of all eigenvalues of problem (1), (2).

\section{The inverse spectral problem}

When proving sufficiency in Theorem 3, we have actually used an effective algorithm for solving the inverse eigenvalue problems (1), (2). Let us formulate this algorithm.

Assume we have an ordered two-sided sequence $\Lambda=\left\{\lambda_{j}\right\}_{j=-\infty}^{\infty}$ of real numbers (22) satisfying conditions 1 and 2 of Theorem 3. In order to find a potential $v$ for problem (1), (2) such that the spectrum of problem (1), (2) would coincide with a given sequence $\Lambda$, we can apply the following algorithm.

Step 1. Using the eigenvalues $\Lambda=\left\{\lambda_{j}\right\}_{j=-\infty}^{\infty}$ construct the characteristic function $\chi(z)$ by formula (21),

$$
\chi(z)=\prod_{k=1}^{\infty}\left(k-\frac{1}{2}\right)\left(\lambda_{k}-z\right)\left(z-\lambda_{-k}\right) .
$$

Step 2. Calculate the values $\chi(n), n \in Z$.

Step 3. The functions $v \in L_{2}(-\pi, \pi)$ will be the ones we are looking for if and only if all their Fourier coefficients will satisfy the identities

$$
\left|1-2 \pi i(-1)^{n} v_{n}\right|^{2}=(-1)^{n} \chi(-n) .
$$

Step 4. By solving the algebraic quadratic equations (26), we find Fourier coefficients $v_{n}$. The potentials are defined in terms of their Fourier series $v(x)=\sum_{n=-\infty}^{\infty} v_{n} e^{i n x}$. 
Example 1. Let us find the potential $v(x)$ for problem (1), (2) from the spectrum $\lambda_{1}=\lambda_{-1}=0$, $\lambda_{n}=n-\frac{1}{2}, \lambda_{-n}=-n+\frac{1}{2}, n=2,3, \ldots$

Step 1. The characteristic function has the form

$$
\chi(z)=\frac{z^{2}}{z^{2}-\frac{1}{4}} \cos \pi z .
$$

Step 2. From (27), calculate the values $\chi(n), n \in Z$,

$$
\chi(n)=\frac{n^{2}}{n^{2}-\frac{1}{4}}(-1)^{n} .
$$

Step 3. Substituting the value of $\chi(n)$ from (28) into (26) we obtain a quadratic equation for $v_{n}$,

$$
\left|1-2 \pi i(-1)^{n} v_{n}\right|^{2}=1+\frac{1}{4 n^{2}-1} .
$$

A solution that has the least norm is the following:

$$
v_{n}=\frac{i(-1)^{n}}{4 \pi\left(4 n^{2}-1\right)}-\frac{i \delta_{n}}{2}, \quad \text { where } \quad \delta_{n}=-\frac{(-1)^{n}}{2 \pi\left(4 n^{2}-1\right)^{2}}\left[1+\sqrt{1+\frac{1}{4 n^{2}-1}}\right]^{-2} .
$$

Step 4. Using the Fourier coefficients we construct the potentials

$$
i v(x)=\frac{1}{4 \pi}+\frac{1}{8} \cos \frac{x}{2}+\sum_{n=1}^{\infty} \delta_{n} \cos n x .
$$

Let $v(x)=\overline{v(x)}$ be a real potential. Then the Fourier series $v(x)=\sum_{n=-\infty}^{\infty} v_{n} e^{i n x}$ can be written as

$$
v(x)=\sum_{n=0}^{\infty}\left(v_{c, n} \cos n x+v_{s, n} \sin n x\right)
$$

with real Fourier coefficients $v_{c, n}, v_{s, n}$. The Fourier coefficients $v_{n}$ with respect to the system $\left\{e^{i n x_{3}}\right\}$ and the Fourier coefficient $v_{c, n}, v_{s, n}$ satisfy the relations $v_{c, n}=v_{n}+v_{-n}, v_{s, n}=i\left(v_{n}-\right.$ $\left.\overline{v_{-n}}\right), v_{n}=\frac{1}{2}\left(v_{c, n}-i v_{s, n}\right)$. Using these relations we get from (10) that

$$
v_{s, n}=\frac{1}{4 \pi}(\chi(n)-\chi(-n)), v_{c, n}^{2}+v_{s, n}^{2}=\frac{1}{2 \pi}\left((-1)^{n}(\chi(n)+\chi(-n))-2\right) .
$$

Corollary 1. A real odd potential $v \in L_{2}$ is defined uniquely by spectrum of problem (1)-(2).

Indeed, for an odd potential, we have that $v_{c, n}=0$. Since the characteristic function $\chi$ is uniquely defined by the spectrum of problem (1)-(2), formulas (30) explicitly give the value of $v_{s, n}$. Then the potential is given by its Fourier series (29). 


\section{Isospectral nonlocal potentials}

Let $\Lambda(\nu)=\left\{\lambda_{j}\right\}_{j=-\infty}^{\infty}$ be an ordered two-sided sequence of eigenvalues of problem (1), (2) with a nonlocal potential $v$. Two potentials $v^{(1)}$ and $v^{(2)}$ are called isospectral if $\Lambda\left(v^{(1)}\right)=$ $\Lambda\left(v^{(2)}\right)$. It follows from Theorem 2 that potentials $v^{(1)}$ and $v^{(2)}$ are isospectral if and only if the corresponding characteristic functions coincide. Using representations (10) for the characteristic functions we obtain a criterion for two potentials $v^{(1)}$ and $v^{(2)}$ to be isospectral in terms of their Fourier coefficients $v_{n}^{(j)}, j=1,2, n \in Z$,

$$
\left|1-2 \pi i(-1)^{n} v_{n}^{(1)}\right|=\left|1-2 \pi i(-1)^{n} v_{n}^{(2)}\right|, \quad \forall n \in Z .
$$

This condition has a simple geometric interpretation. The Fourier coefficients $v_{n}^{(1)}$ and $v_{n}^{(2)}$ of an isospectral potential lie on the same circle in the complex plane with center at the point $c_{n}=\frac{i(-1)^{n+1}}{2 \pi}$.

These considerations easily imply a number of corollaries.

Corollary 2. The set of all potentials that are isospectral to the potential $v(x)$ contains a unique potential $v_{\text {ext }}(x)$ having a minimal $L_{2}$-norm. We also have that

$$
\begin{aligned}
v_{\text {ext }}(x) & =\sum_{n=-\infty}^{\infty} v_{\text {ext }, n} e^{i n x}, \\
v_{\text {ext }, n} & =\frac{i(-1)^{n}}{2 \pi}\left(1-\left|1-2 \pi i(-1)^{n} v_{n}\right|\right),
\end{aligned}
$$

where $v_{n}$ are Fourier coefficients of the potential $v(x)=\sum_{n=-\infty}^{\infty} v_{n} e^{i n x}$.

Consider a complex-valued potential $v(x)$ having the properties of

1. CE-symmetry, $v(x)=\overline{v(-x)}$,

2. CO-symmetry, $v(x)=-\overline{v(-x)}$.

In the case of the CE-symmetry, the real part of the function $v(x)$ is even, and the imaginary part is odd. The Fourier coefficients $v_{n}$ in the decomposition $v(x)=\sum_{n=-\infty}^{\infty} v_{n} e^{i n x}$ of a CE-symmetric function will be real. The function $v(x)$ will be CO-symmetric if and only if its Fourier coefficients are pure imaginary. Note that the potential $v_{\text {ext }}(x)$ in Corollary 2 is a CO-symmetric function.

Isospectral condition (31) for potentials having the CE or CO symmetries take the form

1. $v_{n}^{(1)}= \pm v_{n}^{(2)}$ for CE-symmetric potentials, and

2. $v_{n}^{(1)}=v_{n}^{(2)}$ or $v_{n}^{(1)}+v_{n}^{(2)}=\frac{i(-1)^{n+1}}{\pi}$ for CO-symmetric potentials. 
Corollary 3. If two CO-symmetric potentials $v^{(1)}$ and $v^{(2)}$, which belong to the space $L_{2}(-\pi, \pi)$, are isospectral, then they differ by a trigonometric polynomial,

$$
v^{(1)}-v^{(2)}=\sum_{k=-K}^{K} \varepsilon_{k} e^{i k x}, \quad K<\infty .
$$

Corollary 4. Assume that a priori it is know that the $L_{2}$-norm of a CO-symmetric potential is less than $\frac{1}{\sqrt{2 \pi}}$. Then this potential is uniquely defined by the spectrum.

In fact, by the Parseval equality, $\|\nu\|_{L_{2}}^{2}=2 \pi \sum\left|v_{n}\right|^{2}$, and it follows from the condition $\|v\|_{L_{2}}<\frac{1}{\sqrt{2 \pi}}$ that all Fourier coefficients of the potential $v$ satisfy the estimate $\left|v_{n}\right|<\frac{1}{2 \pi}$. The isospectrality condition for CO-symmetric potentials yields that the isospectral potentials coincide in a ball $\|v\|_{L_{2}}<\frac{1}{\sqrt{2 \pi}}$.

\section{Acknowledgements}

The author expresses his gratitude to DFG for a financial support of the project DFG BR 1686/2-1.

\section{References}

[1] V. A. Yurko, An inverse problem for integro-differential operators, Mat. zametki, 50 (1991), $134-146$ (in Russian) V. A. Yurko, Math. Notes, 50 (1991), 1188-1197 (Engl. transl.)

[2] M. M. Malamud, Similarity of Volterra operators and related questions of the theory of differential equations offractional order, Trans. Moscow Math. Soc., 55 (1994), 57-122.

[3] K. V. Kravchenko, On differential operators with nonlocal boundary conditions, Diff. Uravn., 36 (2000), 464469 K. V. Kravchenko, Diff. Eqns 36(2000), 517-523 (Engl. transl.)

[4] S. A. Buterin, On an inverse spectral problem for a convolution integro-differential operator, Results Math., 50 (2007), 173-181.

[5] S. A. Buterin, On recovering a convolution perturbation of the Sturm Liouville operator from the spectrum, Diff. Uravn. 46 (2010), 146-149 (in Russian) S. A. Buterin, Diff. Eqns , 46 (2010), 150-154 (Engl. transl.)

[6] Yu. V. Kuryshova, An inverse spectral problem for integro-differential operators, Mat. zametki, 81 (2007), 855866 (Russian)Yu. V. Kuryshova, Math. Notes 81 (2007), 767-777 (Engl. transl.)

[7] S. Albeverio and L. P. Nizhnik, Schrödinger operators with nonlocal point interactions, J. Math. Anal. Appl., 332 (2007), 884-895.

[8] S. Albeverio, R. Hryniv and L. Nizhnik, Inverse spectral problems for nonlocal Sturm-Liouville operators, Inverse Problems, 23 (2007), 523-535.

[9] L. P. Nizhnik, Inverse eigenvalue problems for nonlocal Sturm-Liouville operators, Methods Funct. Anal. Topology, 15 (2009), 41-47.

[10] L. P. Nizhnik, Inverse nonlocal Sturm-Liouville problem, Inverse Problems, 26 (2010), 125006.

[11] V. A. Marchenko, Sturm-Liouville Operators and Their Applications, Naukova Dumka Publ., Kiev, 1977 (in Russian); Engl. transl.: Birkhäuser Verlag, Basel, 1986.

Institute of Mathematics, 3 Tereshchenkivska, Kiev 01601, Ukraine.

E-mail:nizhnik@imath.kiev.ua 